\title{
Focus on Staff
}

\section{ASCO Undertakes Oncology Workforce Study}

Will there be a shortage of oncologists in 2015? This is a question that needs to be answered now in order to design and promote programs and policies that can help assure that Americans have access to high quality cancer care in the future. All signs point to a significant increase in cancer rates as the baby boomers reach 65 years of age and older. ${ }^{1}$ Additionally, the 5-year survival rate has risen to $79 \%$ for children and $64 \%$ for adults ${ }^{2}$ and will likely lead to a significant rise in individuals with a need for continuing care and observation. Yet, the production of new clinical oncologists has remained fairly constant (400 to 500 per year $^{3}$ ) over the last decade and we do not know how many of today's practicing oncologists will still be seeing patients in 2015. While there seems to be growing interest in clinical oncology fellowships among medical students and residents, ${ }^{4}$ we do not know whether the numbers of training/fellowship positions will produce enough clinical oncologists to meet future needs.

In order to conduct a comprehensive assessment of the current and likely future supply and demand for practicing oncologists, ASCO has contracted with the Association of American Medical Colleges' (AAMC) Center for Workforce Studies.

One important part of the study is a survey of several thousand clinical oncologists that will be conducted over the next few months. If you are selected for the study, we urge you to complete the survey.

The Center is conducting the study in cooperation with the ASCO Workforce in Oncology Task Force, chaired by Michael Goldstein, MD (medical oncologist, Beth Israel Deaconess Medical Center, Boston). The Task Force includes leaders representing a number of areas: program directors, oncology fellows, health services researchers, community oncologists, academic oncologists, and physician workforce specialists.

The 18-month study will focus on medical oncologists, hematologists/oncologists, gynecologic oncologists, and pediatric hematologists/oncologists. The study will include a detailed analysis of existing data on the incidence of cancer and current utilization patterns and will incorporate new knowledge on practice patterns and career expectations that will be gathered from surveys of practicing clinical oncologists, program directors, fellows completing training, and new entrants in fellowship programs. Information gathered from the study will be used to model future supply and demand. The study contains four main areas of focus: assessment of the supply and distribution of the oncologist workforce, including geographic and demographic data on oncologists currently in practice and a survey of attitudes of practicing oncologists regarding factors influencing physician supply and productivity; assessment of oncologists in training, including demographic data for applicants to fellowship programs and surveys of fellows entering and graduating from fellowship programs and program directors to determine initial career choices and expectations; analysis of existing oncology data, including age-specific cancer incidence rates, population projections and Medicare claims data; and projection of future oncologist workforce needs, taking into account projected cancer incidence and prevalence and adjusting for factors influencing oncologist supply and productivity.

The study will be completed by June 2006, with preliminary results presented at the ASCO Annual Meeting (June 2-6, 2006, Atlanta, Georgia). The survey data will continue to be available to the AAMC Center for Workforce Studies, which may use it to conduct ongoing focused research projects. For additional information on the study, please contact Suanna Bruinooge in ASCO's Cancer Research Department at bruinoos@asco.org.

\section{References}

1. U.S. Centers for Disease Control and Prevention. United States Cancer Statistics; 2000 Incidence Report

2. U.S. Centers for Disease Control and Prevention. Morbidity and Mortality Weekly Report 53:528, 2005

3. JAMA Medical education issues, 1992-2003

4. Todd RF, Gitlin SB, Burns LJ: Subspecialty training in hematology and oncology, 2003: Results of a survey of training program directors conducted by the American Society of Hematology. Blood 103, June 15,2004 